\title{
UAV Path Planning System Based on 3D Informed RRT* for Dynamic Obstacle Avoidance
}

\author{
Jiawei Meng ${ }^{1}$, Sebastian $\mathrm{Kay}^{2}$, Angran $\mathrm{Li}^{2}$, and Vijay M. Pawar ${ }^{1}$
}

\begin{abstract}
A path planning system based on the Informed RRT* path planner was developed to enable an unmanned aerial vehicle (UAV) to avoid moving obstacles in a cluttered 3D environment. For congested environments such as a construction site, path planning systems that help a UAV to safely manoeuvre around dynamic objects and potential co-workers operating within the same workspace is needed. Instead of using a general RRT* path planner approach which will generate a sinuous path, we proposed a flexible approach to increase the convergence of our path planner by re-defining the search space based on 2D Informed RRT* path planner. General RRT* has a relatively low convergence speed to optimize its original solution. By using motion tracking cameras, we obtained real-time feedback of the UAVs pose as well as map structuring and obstacle positions. With this setup, the performance of our proposed path planning approach was assessed using a set of diverse scenarios to compare against general RRT* in convergence rate, quality of solution and ability to handle multiple obstacle situation.
\end{abstract}

\section{INTRODUCTION}

Using swarms of unmanned aerial vehicles (UAVs) to assist and capture information to aid co-working across a large volume is widely being explored across a range of sectors [1]. For example, the potential benefits of using UAV's within a construction site include: reduced construction time via continuous monitoring, in addition to supporting safer construction methods in hard-to-access and dangerous conditions such as working at height [2] and post-disaster reconstruction [3]. Construction sites are interesting environments for testing UAV capabilities due to their scale and need to have other actors co-working within the same working volume; making protocols for safe operation a critical requirement. Further, automation of these protocols is desirable to cope with the complexity of tasks being performed concurrently, and the ability to use these methods whilst

${ }^{1}$ V. M. Pawar, Autonomous Manufacturing Lab, Department of Computer Science, University College London, Gower Street, London, WC1E 6BT, UK (corresponding author to provide phone: +44 (0)20 7679 3698; fax: +44 (0)20 7387 1397; e-mail: v.pawar@cs.ucl.ac.uk).

${ }^{1} \mathrm{~J}$. Meng was with the department of Computer Science at University College London, London, Gower Street, WC1E 6BT, UK (e-mail: uczljme@ ucl.ac.uk)

${ }^{2} \mathrm{~S}$. Kay is with the department of Computer Science at University College London, London, Gower Street, WC1E 6BT, UK (e-mail: sebastian.kay.15@ ucl.ac.uk).

2 A. Li was with the department of Computer Science at University College London, Gower Street, WC1E 6BT, UK. He is now with the Department of Computer Science, University of Toronto, Toronto, Ontario, Canada (e-mail: angran.li.16@ucl.ac.uk). controlling multiple robotic systems. Therefore, to use UAVs within this context, in the first instance we must consider the implementation of appropriate path planning with quick reactions to handle potential scenarios that occur when working within cluttered and highly dynamic environments.

Rapidly exploring random tree (RRT) is one of the main sampling-based path planners as it has a relatively high speed to generate solution in large and high dimensional search spaces [4]. RRT* obtains the global effective solution by randomly building a space-filling tree and efficiently searching non-convex, high-dimensional spaces. Further, RRT* provides an optimal solution through repeating the search process if the time tends to infinity [5]. However, repeating the search process and asymptotically finding the optimal solution is inefficient and not inconsistent with their single-query nature [6]. Hence, 2D Informed RRT* [7] has been proposed demonstrating improved convergence rate, final solution quality, the ability to find difficult paths decrease in the dependence on state dimension, and the size of the search space by generating an ellipsoidal subset of the planning domain. To extend these results for a 3D environment, a new method to generate 3D subset of path planning domain needs to be defined instead of the ellipsoidal subset in 2D environment as it does not consider the Z-dimension and can only surround the path in X-Y plane.

In this paper we show an effective method to tackle the problem occurred when transforming Informed RRT* path planning from 2D environment to 3D environment. We show that our 3D Informed RRT* path planner with an oblique cylinder subset in 3D space achieves a higher convergence rate and better solution than general RRT* when comparing them in path length and search space during same time interval. We also show that 3D Informed RRT* works more efficiently when dealing with multiply obstacle situations when compared with general RRT*.

In strict assumptions (i.e., no obstacles) 2D Informed RRT* improves the linear convergence to reach the optimal solution by generating an ellipsoidal subset of the planning domain [7]. In 3D Informed RRT*, solution is divided into two situations: 1. initial position is equal with target position in any dimension, the question degenerate to the 2D path planning, path planner generates ellipsoidal subset of previous path planning domain; 2. initial position and target position is not equal in any dimension, path planner generates oblique cylinder subset. Altogether, the supported experiments demonstrate a clear improvement of 3D Informed RRT* 
compared with general RRT* in search space, path length, calculation time and number of branches [8].

To demonstrate 3D Informed RRT* is an effective path planner for UAVs in dynamic environments, this paper is organized as follows. Section II presents a background including problem definition and math analysis about optimizing Informed RRT* path planner in 3D environment. Section III presents the system architecture of our UAV path planning system based on 3D Informed RRT*. Section IV presents the experiments designed for comparing the performance of UAV path planning system based on Informed RRT* and RRT* in 3D environment. Section V concludes the paper with a discussion of the path planing system and some related ongoing work.

\section{BACKGROUND}

\section{A. Problem Definition}

The definition of this path planning optimizing problem is similar to S. Karaman and E. Frazzoli [9]. In the definition of [9] and our problem, let $X \subseteq R^{n}$ be the n-dimensional space for our path planning problem. Let $X_{o b s} \subseteq X$ be the space in which the UAV will collide with obstacles. We should pay attention to the change of $X_{o b s}$. As the path planning space should be collision free so $X_{\text {free }}=X \backslash X_{o b s}$. Let the initial position and target position of our UAV belong to the collision free space $X_{\text {strat }} \in X_{\text {free }}$ and $X_{\text {target }} \in X_{\text {free. }}$

Except the space state, we defined an optimal path $\sigma^{*}$ which optimizes the cost function $\mathrm{c}$ and generates a feasible path between $X_{\text {strat }}$ and $X_{\text {target }}[10]$ :

$\sigma^{*}=\arg \min \left\{c(\sigma) \mid \sigma(0)=X_{\text {strat }}, \sigma(1)=X_{\text {target }}, \forall s \in[0,1]\right.$, $\left.\sigma(s)=X_{\text {free }}\right\}$, where $R \geq 0$ is the set of non-negative real numbers.

Then $f(x)$ is defined as the global cost of the path between the initial position and target position at the same time pass the position $x$. The subset which can optimize the current path is defined as:

$$
X_{f}=\left\{x \in X \mid f(x)<c_{\text {best }}\right\} .
$$

Informed RRT* is focusing on increase the convergence rate which can be transform into increasing the probability of adding a random state from $X_{f}[10]$. Because $f(\cdot)$ is unknown in common situation, we use a heuristic function $\hat{f}(\cdot)$ as an estimate for our optimizing problem. Informed RRT* path planner calculates $X_{\hat{f}}$ explicitly and samples from it directly [9]. Through this way, Informed RRT* could work effectively without consider the size of planning problem.

\section{B. $3 D$ Informed $R R T^{*}$}

The ellipsoidal informed subset in 2D Informed RRT* has been proved to improve the convergence rate of the subset in [11]. In this paper, we proposed an effective way to extend the ellipsoidal informed subset from 2D into 3D. Inspired by 2D Informed RRT* directly sample in the ellipsoidal subset, directly sample in an oblique ellipsoid or an oblique cylinder can be feasible. The formulation of defining an oblique ellipsoid has high complexity as it will change the definition of standard coordinate system. The most important reason we choose the oblique cylinder as the subset is because oblique cylinder can compress the information from 3D into $2 \mathrm{D}$. Compressing the information from $3 \mathrm{D}$ in $2 \mathrm{D}$ means only using the information of the path at any height to define the top and bottom surface of the oblique cylinder. Then this method will extend the surface along with the connection of start position and goal position. Through this way, the complexity of defining the subset has been decreased. An example to shown oblique cylinder has better performance than oblique ellipsoid in some specific situation is shown in Fig.1. In this specific situation the subset of oblique cylinder will be smaller than oblique ellipsoid. There is a simple inference based on this example -- oblique ellipsoid will be a good choice when the path has higher complexity at the middle part and lower complexity at the part near the edge.
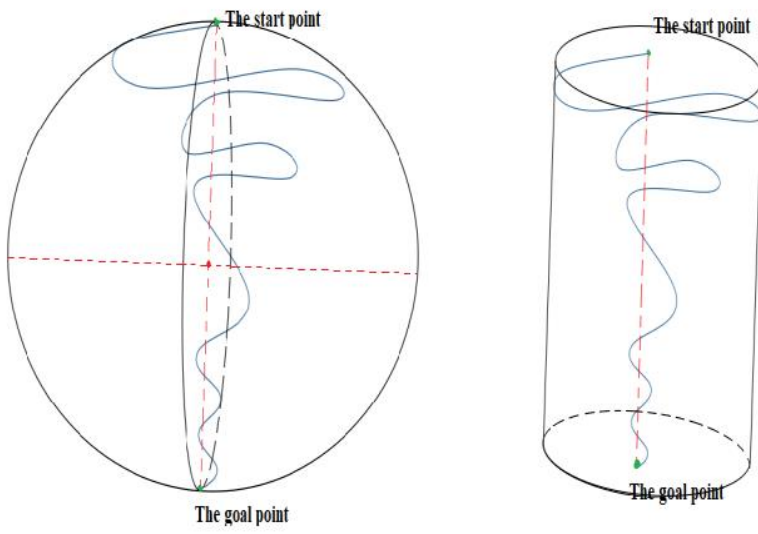

Figure 1. One situation of using oblique ellipsoid and oblique cylinder to define subsets for the same path. The size of these subsets has been roughly shown.

In a 3-dimensional space, a path can be surrounded by a quadratic surface including cylindrical surface, conical surface, spherical surface, ellipsoid, hyperbolic and others. For the situation in Fig.1 we can infer that an inverted conical surface could be a good subset for it. So analyzing the spatial density distribution and defining a classifier which could confirm the best shape of the specific subset and give the method to define these subset. This method can be the optimal solution for optimizing subset of RRT* in 3D space and will also converge the search space at the fastest speed. However, this needs to create a perfect mathematical classifier model with validation [12].

The 3D Informed RRT* path planner implemented by us defines the subset as an oblique cylinder, it could apply to various paths with different spatial probability density distribution. Meanwhile, the oblique cylinder subset has a relatively high convergence rate with an acceptable complexity. The oblique cylinder subset based on standard coordinate system is shown as follows: 


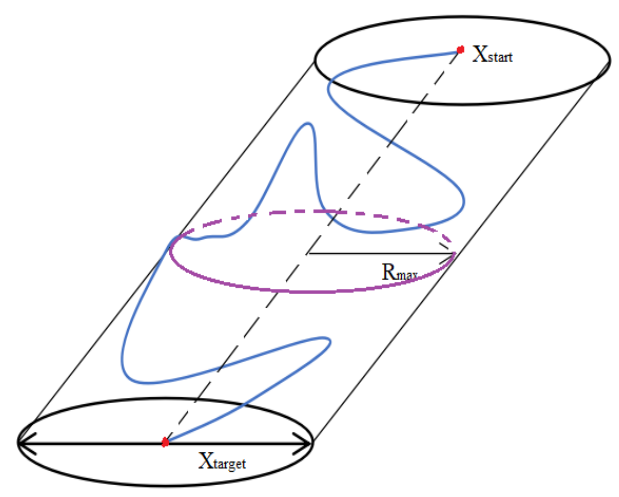

Figure 2. The subset (oblique cylinder space) of 3D Informed RRT*

We assume that the $X_{\text {start }}$ and $X_{\text {target }}$ are not equal in any dimension so the common situation is similar to Fig.2. When $X_{\text {start }}$ and $X_{\text {target }}$ are equal with each other at any dimension in the space, the question will degenerate to $2 \mathrm{D}$ path planning question so we implemented 2D Informed RRT* in the system. The connection of $X_{\text {start }}$ and $X_{\text {target }}$ can define $R_{\max }$ and $R_{\min }$ by measuring the largest and smallest Euclidean distance from the connection to the points on the fundamental path through equation,

$R_{\text {max }}=\arg \max \left\{\rho(\sigma(x)) \mid \sigma(0)=X_{\text {strat }}, \sigma(1)=X_{\text {target }}\right\}$, where $x$ is on the fundamental path.

The equation of defining the shortest distance from the path to the connection is similar:

$R_{\text {min }}=\arg \min \left\{\rho(\sigma(\mathrm{x})) \mid \sigma(0)=X_{\text {strat }}, \sigma(1)=X_{\text {target }}\right\}$, where $x$ is on the fundamental path.

As a result, we obtained the longest distance and shortest distance of an ellipse in X-Y flat. The area of the subset in 3D Informed RRT* is based on the subset in X-Y flat which has been implemented in 2D Informed RRT* and along with the connection of $X_{\text {start }}$ and $X_{\text {target }}$. Then Informed RRT* algorithm in 3-dimensional space directly informs subset to sample. This path planner searches for optimal path $\sigma^{*}$ by incrementally building a tree in $X_{\text {free }}$ [13], which is the same as RRT* path planner. But it differs from RRT* once the fundamental solution is found and differs from 2D Informed RRT* in the part of defining subset space [13].

\section{SySTEM ARCHITECTURE}

\section{A. Hardware System Architecture}

In this paper we used an OptiTrack 12 camera motion tracking system achieving a $5 \times 5 \times 5$ cubic meter space for the following experiments. Markers are installed on every object inside the experiment site with portraying the profile of it such as our UAVs and obstacles. The obstacles in this experiment are cylinders and are fixed on AGVs controlled by different joysticks. As a result, the real-time pose of each object inside the experiment site can be detected.

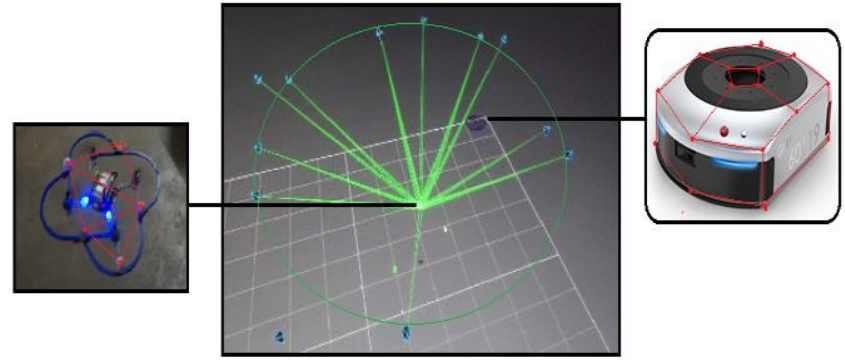

Figure 3. The middle figure shows the interface of tracking system. The left figure shows UAV with markers. The right figure shows dynamic obstacle dynamic obstacle (AGV) with markers on them to portray their profile.

\section{B. Software System Architecture}

This part controls the process of all the built-in algorithms such as 3D Informed RRT* and RRT* based on Robot Operating System in Linux environment [14]. Moreover, we used Rviz (a visualization tool) to visualize the path planning process and monitor the situation of the real path by communicating with UAV through bluetooth. The command for UAV to take off is sent from a controller through bluetooth. The flying UAV will hover at the last position when the path planner is calculating the new path. The command for UAV to land on the ground will be executed once the real-time path planning is finished.

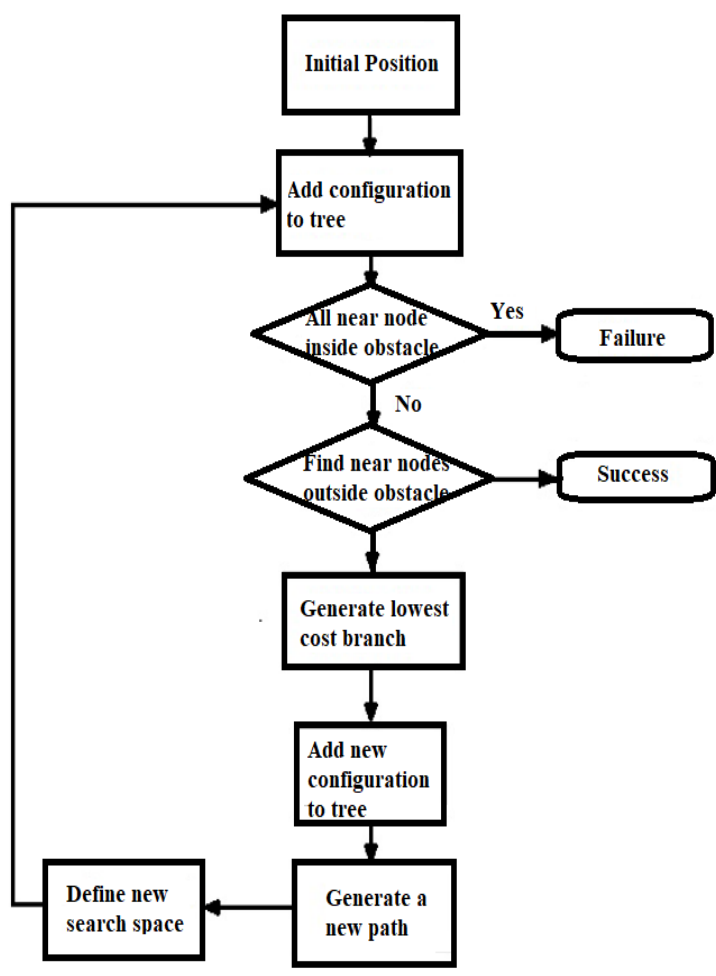

Figure 4. The process of 3D Informed path planner generates path for the UAV-based path planning system 


\section{EXPERIMENT DESIGN AND RESULt ANALYSIS}

In the experiment, 3D Informed RRT* is compared with $\mathrm{RRT}^{*}$ in a variety of path planning situations $(1,3$ and 6 dynamic obstacles in the map). 3D Informed RRT* and RRT* were given 5.0 seconds to optimize their solution and the experiments focus on the convergence rate and the performance of global optimizing the path. When measuring parameters to reflect the performance, each parameter has been recorded 150 times based on 150 running times of each experiment for these path planners. The paths of both path planners in each running time are given to real UAVs in the laboratory to fly and avoid obstacles. As the real flight data is influenced by the factors such as the accuracy of control and battery power, the performance of the path planners will be reflected based on simulation data.

\section{A. Experiment Design}

This experiment is in cubic space where $l$ equals to 5 meters and $w$ equals to 0.2 meters. The positions of $X_{\text {start }}$ and $X_{\text {goal }}$ are constant in the map and the top view of the map is organized as follows:

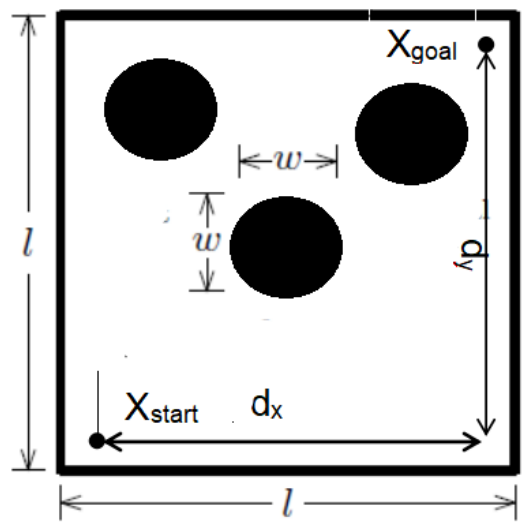

Figure 5. The top view of the UAV path planning experiment for 3 dynamic obstacles. The map of 1 dynamic obstacle and 6 dynamic obstacles can refer to this map.
RRT*

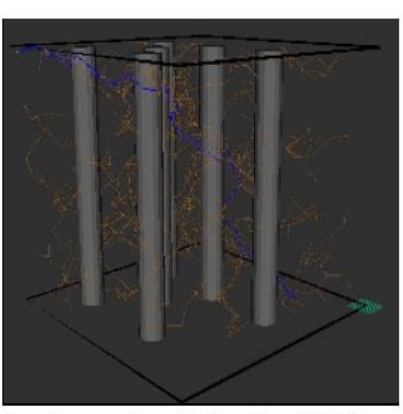

$\mathrm{t}=5$ seconds, path length $=18.13$
Informed RRT*

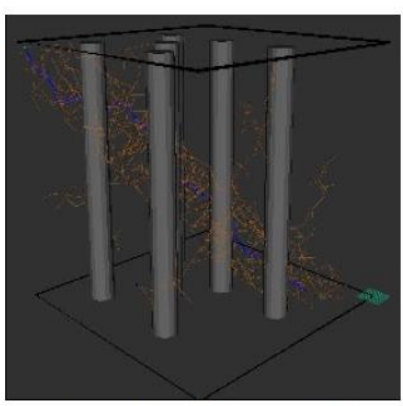

$\mathrm{t}=5$ seconds, path length $=13.95$
Figure 6. A recording for real-time feedback of the path and search space in 6 dynamic obstacles condition. The figure at the left belongs to RRT* and the figure at the right belongs to Inform RRT*. The blue line is the optimized solution and orange part is the search space.
Longitudinal comparing the result of $\mathrm{RRT}^{*}$ and 3D Informed RRT* from 1 to 3 and then to 6 dynamic obstacles based on the following table (parameters including path length to reflect the quality of the latest solution and the size of search space to reflect the convergence rate):

TABLE I. PARAMETER RESUlt OF DIFFERENT OBSTACLE NUMBER

\begin{tabular}{|c|c|c|}
\hline \multicolumn{3}{|c|}{$\begin{array}{l}\text { Path planning with } 1 \text { dynamic obstacle }(t=5.0 \text { second, } \\
\text { repetition }=150)\end{array}$} \\
\hline Name of path planner & $R R T^{*}$ & $\begin{array}{c}\text { Informed } \\
\text { RRT }^{*}\end{array}$ \\
\hline Path length (m) & 14.3 & 13.7 \\
\hline Search space $\left(\mathrm{m}^{3}\right)$ & 125 & 20.4 \\
\hline \multicolumn{3}{|c|}{$\begin{array}{l}\text { Path planning with } 3 \text { dynamic obstacles }(t=5.0 \text { second, } \\
\text { repetition }=150)\end{array}$} \\
\hline Name of path planner & $R R T^{*}$ & $\begin{array}{c}\text { Informed } \\
R_{R T} *\end{array}$ \\
\hline Path length (m) & 15.6 & 14.1 \\
\hline Search space $\left(\mathrm{m}^{3}\right)$ & 125 & 22.7 \\
\hline \multicolumn{3}{|c|}{$\begin{array}{l}\text { Path planning with } 6 \text { dynamic obstacles }(t=5.0 \text { second, } \\
\text { repetition }=150)\end{array}$} \\
\hline Name of path planner & $R R T^{*}$ & $\begin{array}{c}\text { Informed } \\
R_{R} T^{*}\end{array}$ \\
\hline Path length (m) & 17.3 & 14.3 \\
\hline Search space $\left(\mathrm{m}^{3}\right)$ & 125 & 31.3 \\
\hline
\end{tabular}

TABLE II. SOLUTION IMPROVEMENT IN INFORMED RRT**

\begin{tabular}{|c|c|c|}
\hline $\begin{array}{c}\text { Informed } \\
\text { RRT }^{*}\end{array}$ & $\begin{array}{c}\text { Path optimization } \\
\text { rate }(\%)\end{array}$ & $\begin{array}{c}\text { Search space in total } \\
\text { space }(\%)\end{array}$ \\
\hline 1 obstacle & 4.19 & 16.32 \\
\hline 3 obstacles & 8.97 & 18.16 \\
\hline 6 obstacles & 11.56 & 25.04 \\
\hline
\end{tabular}

\section{B. Result Analysis}

Perfor mance comparison between 2 path planners in 3D space on branch quantity Search space (cubic meter)

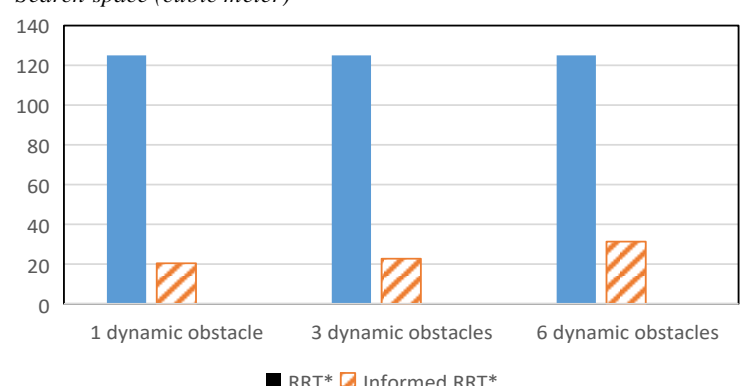

Figure 7. The horizontal comparison of RRT* path planner and Informed RRT* path planner on number of branches under different obstacle condition. 


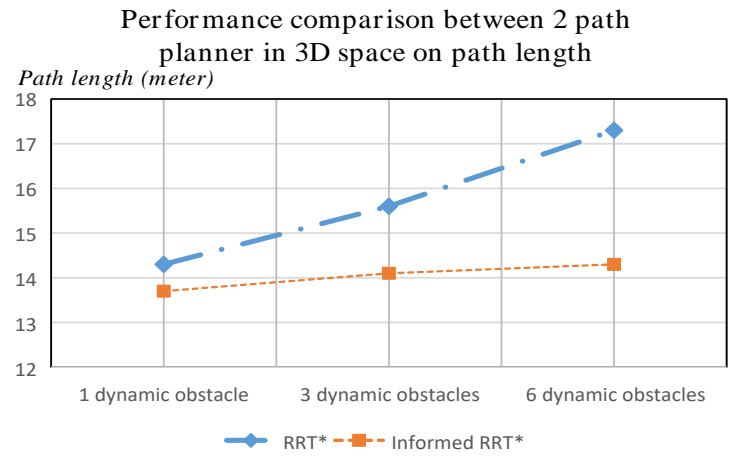

Figure 8. The horizontal comparison of RRT* path planner and Informed RRT $^{*}$ path planner on path length under different obstacle condition.

Based on the experimental result of Fig.7, we can see the search space of 3D Informed RRT* is $16.32 \%, 18.16 \%$ and $25.04 \%$ of the search space of general RRT*. These path planner begin with the same unoptimized original condition. Experiments in various number of moving obstacles show that 3D Informed RRT* is capable of obtaining near-optimal solutions in significantly smaller search space than RRT* [7]. Informed RRT* path planner has a lower cost than RRT* path planner in 3D space when the computation time is equal. Re-defining subset as a smaller space could increase the convergence rate of RRT* path planner. With the repetition increased, the search space keeps decreasing of Informed RRT* compared with general RRT*. In another word, 3D Informed RRT* more likely to find the optimal path under limited time constraint.

Based on the observation of Fig.8, 3D Informed RRT* has significant improvement than general RRT* in solutions when the varying the number of moving obstacles. The path reduced $4.19 \%(0.6 \mathrm{~m}), 8.97 \%(1.4 \mathrm{~m})$ and $11.56 \%$ (2.0 m) in Informed RRT* than in general RRT*. The performance improvement in 3D Informed RRT* is outstanding in cluttered environment. In cluttered environment, the probability of generating small gaps grows rapidly with incremental dynamic obstacles. As a result, another experiment can be implemented in the future to measure the ability of 3D Informed RRT* when sampling with difficult passages (this situation is more likely to happen when the quantity of obstacles is huge) [7]. In addition, a hypothesis can be derived as follows, the larger quantity of obstacles in the map, the better performance 3D Informed RRT* will have compared with RRT*. As [6] has proved that Informed RRT* has the ability restrict the search space and regardless of the size of path planning problem.

\section{CONCLUSION}

In our experiments, we show that 3D Informed RRT* trend to generate fewer branches in the newly defined search space. We also demonstrate that 3D Informed RRT* can easily handle problems with lots of obstacles. It can be viewed as a technique to generate open-loop path for nonlinear system with state constraints.

The research about the path planning method presented in this paper is able to combine with real applications as it can rapidly optimizing a solution for UAV. There are several lines of research arising from the UAV path planning system. Rapid path planning can also be used in building task and it is not only used in obstacle avoidance while carrying materials [15]. An open path planning code implemented of Informed RRT* in $3 \mathrm{D}$ is described at https://github.com/jiaweimeng.

\section{ACKNOWLEDGMENT}

This research was funded by contributions from the Autonomous Manufacturing Lab at University College London under the department of Computer Science. Furthermore, I would like to acknowledge Dr. Vijay M. Pawar who is my supervisor and the director of this project, Sebastian Kay who contributes to the UAV control system and tracking system and Angran Li who is my colleague with working on various path planning method for UAV-based system.

\section{REFERENCES}

[1] N. Ahmed and J. Page, "Manufacture of an Unmanned Aerial Vehicle (UAV) for Advanced Project Design Using 3D Printing Technology", Applied Mechanics and Materials, vol. 397-400, pp. 970-980, 2013.

[2] W. Zhang and J. Wu, "To Explore the UAV Application in Disaster Prevention and Reduction", Applied Mechanics and Materials, vol. 590, pp. 609-612, 2014

[3] S. Siebert and J. Teizer, "Mobile 3D mapping for surveying earthwork projects using an Unmanned Aerial Vehicle (UAV) system", Automation in Construction, vol. 41, pp. 1-14, 2014.

[4] L. Gong, Y. Zhang and J. Cheng, "Coordinated Path Planning Based on RRT Algorithm for Robot", Applied Mechanics and Materials, vol 494-495, pp. 1003-1007, 2014.

[5] B. Feng and Y. Liu, "An Improved RRT Based Path Planning with Safe Navigation", Applied Mechanics and Materials, vol. 494-495, pp. 1080-1083, 2014.

[6] J. Nasir, F. Islam, U. Malik, Y. Ayaz, O. Hasan, M. Khan and M. Muhammad, "RRT*-SMART: A Rapid Convergence Implementation of RRT*”, International Journal of Advanced Robotic Systems, vol. 10, no. 7, p. 299, 2013.

[7] J. Gammell, T. Barfoot and S. Srinivasa, "Informed Sampling for Asymptotically Optimal Path Planning", IEEE Transactions on Robotics, pp. 1-19, 2018

[8] C. Moon and W. Chung, "Kinodynamic Planner Dual-Tree RRT (DT-RRT) for Two-Wheeled Mobile Robots Using the Rapidly Exploring Random Tree", IEEE Transactions on Industrial Electronics vol. 62, no. 2, pp. 1080-1090, 2015.

[9] S. Karaman and E. Frazzoli, "Sampling-based algorithms for optimal motion planning", The International Journal of Robotics Research, vol. 30, no. 7, pp. 846-894, 2011.

[10] N. Tokuse, H. Sakahara and F. Miyazaki, "Motion Planning which Produces a Give-way Behavior using Spatiotemporal RRT", Journal of the Robotics Society of Japan, vol. 27, no. 6, pp. 696-701, 2009.

[11] L. CAO, Y. JIA and A. ZHANG, "Path planning for multiple unmanned combat aerial vehicles based on improved artificial bee colony algorithm", Journal of Computer Applications, vol. 33, no. 12, pp 3596-3599, 2013.

[12] H. Chiang and L. Tapia, "COLREG-RRT: An RRT-Based COLREGS-Compliant Motion Planner for Surface Vehicle Navigation", IEEE Robotics and Automation Letters, vol. 3, no. 3, pp. 2024-2031, 2018

[13] D. MO and G. LIU, "Improved RRT-Connect path planning algorithm for biped robot", Journal of Computer Applications, vol. 33, no. 8, pp. 2289-2292, 2013

[14] N. Gasilov, M. Dogan, and V. Arici, "Two-stage shortest path algorithm for solving optimal obstacle avoidance problem," IETE Jour. Of Research, 57(3): 278-285, May 2011.

[15] E. Plaku, "Region-Guided and Sampling-Based Tree Search for Motion Planning With Dynamics", IEEE Transactions on Robotics, vol. 31, no. 3 , pp. 723-735, 2015 . 